\section{Regelmäßige Screenings verbessern Verlauf bei Uveitis}

Walscheid K et al. Adherence to ophthalmological screening recommendations and course of uveitis in children with juvenile idiopathic arthritis: data from the Inception Cohort of Newly diagnosed patients with JIA (ICON-JIA) study. Clin Exp Rheumatol 2020; 38: 792-798

Die juvenile idiopathische Arthritis (JIA) ist die häufigste rheumatische Erkrankung bei Kindern. Die Früherkennung einer beginnenden Uveitis (JIAU) mit sofortiger Therapie ist von großer Bedeutung, um eine gute Sehprognose zu gewährleisten. Daher werden augenärztliche Routineuntersuchungen für alle JIA-Patienten empfohlen. Walscheid et al. analysierten die Krankheitsmerkmale bei JIAU in Abhängigkeit von der Einhaltung der Screeningintervalle.

Besonders Hochrisikopatienten halten sich oft nicht an die empfohlenen engmaschigen Screeningintervalle, was zu einer verminderten Sehschärfe bei der Erstdiagnose einer Uveitis führt. In diese Studie schlossen die Wissenschaftler der deutschen Inzeptionskohorte ICON Patienten mit einer JIADiagnose nach ILAR-Kriterien und einer Krankheitsdauer von weniger als 12 Monaten ein. Eine detaillierte Dokumentation der klinischen Daten und Laborparameter führten die Experten zunächst bei der Studienrekrutierung und dann alle 3 Monate während des ersten Jahres der Nachbeobachtung und danach alle 6 Monate durch. Jedem JIA-Patienten, der in die ICON-Studie eingeschlossen wurde, empfahlen die Wissenschaftler in Übereinstimmung mit den aktuellen Screening-Richtlinien in Deutschland augenärztliche Vorsorgeuntersuchungen. Bei jeder Studienvisite befragten die Forscher die Eltern, ob Untersuchungen stattgefunden haben dokumentierten die Besuchsdaten. Ein spezialisierter Augenarzt untersuchte jene Kinder, die zwischenzeitlich eine Uveitis entwickelten. Eine Uveitis klassifizierten die Wissenschaftler nach SUN-Kriterien.

Die Experten schlossen insgesamt 557 Kinder in die Studie ein, von denen 46 (8,3\%) eine Uveitis entwickelten. 63,9\% der Patienten befolgten die empfohlenen Screening-Intervalle. Die Befolgung der Screening-Empfehlungen korrelierte nicht mit dem sozioökonomischen Stress der Familien. Die Forscher beobachteten jedoch einen Zusammenhang zwischen der Befolgung der Screening-Empfehlungen und einem höheren Alter bei Studieneinschluss, einem höheren Alter bei der JIA-Diagnose und einer längeren Krankheitsdauer. Zudem war die Sehschärfe bei den Patienten, die regelmäßige Screenings durchführen ließen, im Vergleich besser. Die Patienten, die zu jenen gehörten, die Screening-Besuche versäumten, verpassten im Durchschnitt 2 Besuche, wiesen häufiger antinukleäre Antikörper auf und hatten häufiger eine Oligoarthritis. Abgesehen von diesen Erkenntnissen schien die Befolgung der ScreeningEmpfehlungen keinen Einfluss auf die klinische Präsentation oder den Verlauf der Uveitis zu haben: Weder war der Schweregrad der Uveitis bei der Erstdiagnose unterschiedlich, noch unterschied sich der Krankheitsverlauf während der 3-jährigen Nachbeobachtungszeit signifikant.

\section{FAZIT}

Das Screening auf Uveitis sollte bei JIA-Patienten auch in Zukunft obligatorisch sein, da eine große Anzahl von JIA-Patienten bereits bei der Erstdiagnose schwere Komplikationen aufweisen. Neuere Erkenntnisse über die Risikofaktoren für die Entwicklung einer Uveitis sowie neue therapeutische Strategien müssen berücksichtigt werden, was im Idealfall zu einem individualisierten Zeitplan für jeden Patienten resultieren könnte, so die Autoren.

Dr. Maddalena Angela Di Lellis, Tübingen 